\title{
Optimal statistical damage detection and classification in an experimental wind turbine blade using minimum instrumentation
}

\author{
Simon Hoell ${ }^{\mathrm{a}} \&$ Piotr Omenzetter ${ }^{*} \mathrm{~b}$ \\ ${ }^{a}$ Institute of Structural Mechanics, Bauhaus University Weimar, Weimar, Germany \\ ${ }^{b}$ The LRF Centre for Safety and Reliability Engineering, The University of Aberdeen, Aberdeen, \\ UK
}

\begin{abstract}
The increasing demand for carbon neutral energy in a challenging economic environment is a driving factor for erecting ever larger wind turbines in harsh environments using novel wind turbine blade (WTBs) designs characterized by high flexibilities and lower buckling capacities. To counteract resulting increasing of operation and maintenance costs, efficient structural health monitoring systems can be employed to prevent dramatic failures and to schedule maintenance actions according to the true structural state. This paper presents a novel methodology for classifying structural damages using vibrational responses from a single sensor. The method is based on statistical classification using Bayes' theorem and an advanced statistic, which allows controlling the performance by varying the number of samples which represent the current state. This is done for multivariate damage sensitive features defined as partial autocorrelation coefficients (PACCs) estimated from vibrational responses and principal component analysis scores from PACCs. Additionally, optimal DSFs are composed not only for damage classification but also for damage detection based on binary statistical hypothesis testing, where features selections are found with a fast forward procedure. The method is applied to laboratory experiments with a small scale WTB with wind-like excitation and non-destructive damage scenarios. The obtained results demonstrate the advantages of the proposed procedure and are promising for future applications of vibration-based structural health monitoring in WTBs.
\end{abstract}

Keywords: damage classification, statistical classification, principal component analysis, wind turbines, time series methods, vibration analysis

\section{INTRODUCTION}

The wind energy sector is subject to continuous advancements due to the increasing public demands for carbon neutral energy in a competitive economic environment. The key methodologies for improving potential energy outputs are increasing the sizes of wind turbines (WTs) and erecting them in remote areas, such as offshore and hill-tops, where wind speeds are higher and more predictable. This requires that WT blades (WTBs) become lighter, which can be achieved by using novel composite materials. However, the resulting higher flexibilities and lower buckling capacities adversely affect long-term safety and reliability ${ }^{1}$. Additionally, rigid intervals for in-situ inspections are defined by international standards and guidelines ${ }^{2,3}$. This practice applied to large WTs in remote sites causes significant operation and maintenance costs, which can make up to $20 \%$ of the total energy production costs ${ }^{4}$. Structural health monitoring (SHM) can make a difference by scheduling maintenance actions according to the true structural state and, with it, balancing the risk of failures with inspection and maintenance costs.

Vibration-based SHM methods have an edge over other available techniques due to the applicability for continuous monitoring of complex structures in harsh environments and their moderate instrumentation efforts. These advantages are results of the availability of mature sensing techniques and utilization of global vibration responses from ambient excitations of environmental sources, such as wind and waves, or operations, e.g. traffic and rotation of machinery. The resulting elastic waves can encompass the entire structure due to long wave length and low damping. Several damage sensitive features (DSFs) can be extracted from such vibrational responses in order to describe the current state of a

* piotr.omenzetter@abdn.ac.uk; phone 44-1224-272529; fax 44-1224-272497; www.abdn.ac.uk 
Return to the Manage Active Submissions page at http://spie.org/submissions/tasks.aspx and approve or disapprove this submission. Your manuscript will not be published without this approval. Please contact author_help@spie.org with any questions or concerns.

structure in a meaningful and efficient manner. Common DSFs are modal parameters ${ }^{5}$, i.e. natural frequencies, mode shapes and their spatial derivatives, and parametric time series representations, e.g. autoregressive models ${ }^{6}$. Nevertheless, identifying appropriate mathematical models and estimating corresponding DSFs is difficult to automate and can be computationally demanding for these approaches. In contrast, the conceptual simplicity and ease of computation makes non-parametric time series representations an attractive alternative. Non-parametric DSFs can be defined in the frequency domain, time-frequency domain or time domain using for example power spectral densities ${ }^{7}$, the Hilbert-Huang transform ${ }^{8}$ or cross-correlation functions ${ }^{9}$, respectively. In the present paper, partial autocorrelation coefficients (PACCs) estimated from signals of a single sensor are selected for defining non-parametric time series based DSF vectors. PACCs are closely related to autoregressive parameters and autocorrelation coefficients, and they can all be interchangeably calculated from one another ${ }^{10}$.

After defining a suitable DSF type, the selection of appropriate procedures for specific SHM tasks is required. Structural damage detection (SDD) is a binary problem of deciding whether a structure is healthy or damage occurred. This problem can be efficiently solved by means of binary statistical hypothesis testing, a simple but robust method, which requires only a priori information about the healthy state. It is therefore chosen for SDD in the present study. For advanced SHM tasks, such as structural damage classification (SDC), a different approach is required in order to incorporate a priori knowledge about distinct damage characteristics of different structural states. Soft-computing techniques allow learning nonlinear mappings of DSFs to damage characteristics from data. Support vector machines were employed for SDC in a numerical bridge structure by Liu and $\mathrm{Jiao}^{11}$. A similar method was proposed for SDC in a three story building by Kim et al. ${ }^{12}$. Artificial neural networks were proposed by Bandara et al. ${ }^{13}$ for damage localization and severity estimation in a numerical two-story building. De Lautour and Omenzetter ${ }^{14}$ applied K-nearest neighbor and learning vector quantization classification in experimental and numerical structures. Although these approaches enable to learn complex relations from data, overfitting and high computational demands due to the so called "curse of dimensionality' are practical hindrances for their application.

A statistical approach is therefore pursued in the present paper, which allows obtaining decisions about the structural state promptly and with high efficiency. Here, statistical classification on the basis of Bayes' theorem is applied for solving the multiclass problem of SDC. In contrast to conventional approaches, where only single DSF samples can be classified, it is proposed to incorporate an advanced test statistic which allows classifying bins of samples, where increasing numbers of samples can improve the classification accuracy. However, independent of the SHM procedure, including noisy and/or insensitive DSF components can alter the performance of an algorithm unfavorably. To reduce uncontrollable effects from varying excitation characteristics and measurement noise, initial DSFs are transformed with the help of principal component analysis (PCA) ${ }^{15}$. Then, fast forward (FF) selection is applied for composing optimized DSF subsets, where components are iteratively added to a DSF vector by maximizing an objective function. The selection of features is studied for PCA scores and original PACCs as a benchmark. The objective function should include the characteristics of an SHM task and algorithm to achieve the best performance. Thus, a function based on binary statistical hypothesis testing is employed for SDD and a measure of classification accuracies is chosen for SDC. The proposed methods are applied to experiments with a small scale WTB made of a glass-fiber reinforced epoxy composite material. Damage is simulated non-destructively by attaching small masses, while the WTB is contact-free excited with the help of a pedestal fan.

The layout of the paper is as follows. First, the theory of optimized SDD and SDC by means of statistical hypothesis testing and classification using PACCs and PCA scores of PACCs is introduced. Second, the small scale WTB experiments are presented including the extraction of initial DSFs. The following two sections discuss the application of approaches for optimized SDD and SDC, respectively. Finally, the paper is rounded up by a set of conclusions.

\section{THEORY}

\subsection{Partial autocorrelation-based damage sensitive features}

The proposed methodology aims to detect and classify structural damages on the basis of discrete vibrational response signals, $x[t]$, where $t$ is the time instant. These signals can be divided into $n$ segments $x_{j}[t]$ with common length $n_{\text {samp }}$. To reduce effects of varying excitation conditions, the time series segments can be normalized by removing estimated means and dividing by estimated standard deviations. For describing the structural state in a meaningful and efficient way, PACCs, $\alpha_{j}[\tau]$, at lag $\tau$ can be calculated from the normalized signals, $z_{j}[t]$, by the following recursion ${ }^{16}$ : 
Return to the Manage Active Submissions page at http://spie.org/submissions/tasks.aspx and approve or disapprove this submission. Your manuscript will not be published without this approval. Please contact author_help@spie.org with any questions or concerns.

$$
\hat{\alpha}_{j}[\tau]=\frac{\hat{r}_{j}[\tau]-\sum_{p=1}^{\tau-1} \hat{a}_{j, \tau-1, p} \hat{r}_{j}[\tau-p]}{1-\sum_{p=1}^{\tau-1} \hat{a}_{j, \tau-1, p} \hat{r}_{j}[p]}
$$

where $\hat{a}_{i, l, p}$ denotes the estimated $l$-th coefficient of an autoregressive model of order $p$, and $\hat{r}_{j}[\tau]$ is the sample autocorrelation function at lag $\tau$ of the $j$-th time series segment. The PACCs describe the correlation between the shifted time series $z_{j}[t]$ and $z_{j}[t-\tau]$ without the effects of intermediate variables $z_{j}[t-1], z_{j}[t-2], \ldots, z_{j}[t-\tau+1]$.

Due to the relationship to autoregressive processes, where PACCs of lags higher than the required autoregressive model order are theoretically zero, an appropriate number of lags for constructing DSF vectors can be selected with respect to their probability distribution. The PACCs at higher lags are approximately zero-mean Gaussian distributed with variance

$$
\sigma_{\hat{\alpha}_{j}[\tau]}^{2}=1 / n_{\text {samp }}
$$

By setting a confidence level, this statistic allows selecting $m$ significant PACCs. Nevertheless, the probability distribution of all PACCs is in general difficult to derive in their initial space, thus Fischer's z-transform is applied for obtaining approximately normally distributed scores, $\hat{\alpha}_{j}^{*}[\tau]$, from PACCs $\hat{\alpha}_{j}[\tau]$ under the assumption of normally distributed time series segments $z_{j}[t]$ as ${ }^{17}$ :

$$
\hat{\alpha}_{j}^{*}[\tau]=\tanh ^{-1}\left(\hat{\alpha}_{j}[\tau]\right)=\frac{1}{2} \ln \left(\frac{1+\hat{\alpha}_{j}[\tau]}{1-\hat{\alpha}_{j}[\tau]}\right)
$$

Then, multivariate DSF vectors, $\boldsymbol{\alpha}_{j}$, can be composed of z-scores of the $m$ significant PACCs as:

$$
\boldsymbol{\alpha}_{j}=\left[\begin{array}{llll}
\hat{\alpha}_{j}^{*}[1] & \hat{\alpha}_{j}^{*}[2] & \cdots & \hat{\alpha}_{j}^{*}[m]
\end{array}\right]^{T} \square \mathrm{N}(\boldsymbol{\mu}, \boldsymbol{\Sigma})
$$

where superscript $T$ denotes transpose. They follow a multivariate Gaussian probability distribution, $\mathrm{N}(\boldsymbol{\mu}, \boldsymbol{\Sigma})$, with mean vector $\boldsymbol{\mu}$ and variance-covariance matrix $\boldsymbol{\Sigma}$.

\subsection{Principal component analysis}

Variations in environmental and operational conditions can adversely affect the identifiability of a structural state. Therefore, PCA can be applied to transform initial DSF vectors, $\boldsymbol{\alpha}_{j}$, to PCA scores, $\mathbf{s}_{j}$. The scores are characterized by linear independence and their variance is maximized in the first few dimensions ${ }^{18}$. This allows separating undesired effects with high variability from other meaningful dimensions. Additionally, under the assumption that these variations are present in all datasets, PCA can be performed using only DSFs from the healthy state. A DSF matrix, A, can be constructed from DSF vectors, $\boldsymbol{\alpha}_{j}$, from $n_{\text {seg }}$ time series segments as:

$$
\mathbf{A}^{T}=\left[\begin{array}{llll}
\left(\boldsymbol{\alpha}_{1}-\hat{\boldsymbol{\mu}}\right) & \left(\boldsymbol{\alpha}_{2}-\hat{\boldsymbol{\mu}}\right) & \cdots & \left(\boldsymbol{\alpha}_{n_{s e g}}-\hat{\boldsymbol{\mu}}\right)
\end{array}\right]
$$

where the estimated mean vector, $\widehat{\boldsymbol{\mu}}$, is removed. The PCA transformation matrix, $\mathbf{T}$, can be obtained by singular value decomposition of the estimated variance-covariance matrix $\widehat{\boldsymbol{\Sigma}}$ of the initial DSFs as ${ }^{18}$ :

$$
\hat{\boldsymbol{\Sigma}}=\mathbf{T} \mathbf{\Lambda} \mathbf{T}^{T} \text { with } \mathbf{T}=\left[\begin{array}{llll}
\mathbf{t}_{1} & \mathbf{t}_{2} & \cdots & \mathbf{t}_{m}
\end{array}\right] \text { and } \boldsymbol{\Lambda}=\operatorname{diag}\left(\lambda_{1}, \lambda_{2}, \ldots, \lambda_{m}\right)
$$

where $\mathbf{t}_{i}$ are the principal components (PCs), and $\boldsymbol{\Lambda}$ denotes a diagonal matrix containing the $m$ eigenvalues, $\lambda_{i}$, sorted in descending order. The PCA scores matrix, $\mathbf{S}$, can be written as:

$$
\mathbf{S}=\mathbf{T}^{T} \mathbf{A} \text { with } \mathbf{S}=\left[\begin{array}{llll}
\mathbf{s}_{1} & \mathbf{s}_{2} & \cdots & \mathbf{s}_{n_{\text {samp }}}
\end{array}\right]
$$

where $\mathbf{s}_{i}$ are the score vectors. 
Return to the Manage Active Submissions page at http://spie.org/submissions/tasks.aspx and approve or disapprove this submission. Your manuscript will not be published without this approval. Please contact author_help@spie.org with any questions or concerns.

\subsection{Fast forward feature selection}

A consecutive selection of DSF components is required for initial DSFs, i.e. the PACCs and PCA scores of PACCs in order to improve the SHM performance. This is herein done using a FF procedure which begins with an iterative ranking of DSF components with respect to a given objective function. In the first iteration, the objective function is evaluated for all individual components and the one with the highest value is retained. Next, the remaining components are added in turn to the selected one and the component leading to the highest objective function value for the now bivariate DSFs is selected. This procedure continues until all components are included in the DSF vector. The rank of a component corresponds to the iteration when it is selected, thus the component identified in the first iteration has rank one. The optimal subset of DSF components is the one that maximizes the objective function over all DSF dimensions.

\subsection{Statistical hypothesis testing}

For deciding whether a structure is healthy or damaged using multivariate Gaussian distributed DSFs, the following statistical hypothesis can be defined on the basis of the true multivariate means from the healthy state, $\boldsymbol{\mu}_{h}$, and the current state, $\boldsymbol{\mu}_{c}$ :

$$
\begin{array}{lll}
H_{0}: & \boldsymbol{\mu}_{c}=\boldsymbol{\mu}_{h} \quad \text { (healthy) } \\
H_{1}: \quad \boldsymbol{\mu}_{c} \neq \boldsymbol{\mu}_{h} & \text { (damaged) }
\end{array}
$$

The null and alternative hypothesis, $H_{0}$ and $H_{1}$, describe the healthy and the damaged state, respectively. The true statistical properties are generally not available in practical applications. Therefore, the $T^{2}$ statistic is employed for testing the above hypothesis using estimated quantities ${ }^{19}$ :

$$
T^{2}=\frac{n_{c} n_{h}}{n_{c}+n_{h}}\left(\hat{\boldsymbol{\mu}}_{c}-\hat{\boldsymbol{\mu}}_{h}\right)^{T} \hat{\boldsymbol{\Sigma}}_{p l}^{-1}\left(\hat{\boldsymbol{\mu}}_{c}-\hat{\boldsymbol{\mu}}_{h}\right) \sim T_{m, n_{c}+n_{h}-2}^{2}
$$

where $n_{h}$ and $n_{c}$ are the sample numbers for estimating the statistical properties in the healthy and the current state, respectively. These estimated quantities are indicated by the hat. The distance between the mean vectors is standardized by the pooled variance-covariance matrix estimate, $\widehat{\boldsymbol{\Sigma}}_{p l}$, which is defined by the unbiased estimates of the healthy and current state variance-covariance matrices, $\widehat{\boldsymbol{\Sigma}}_{c}$ and $\widehat{\boldsymbol{\Sigma}}_{h}$, as:

$$
\hat{\boldsymbol{\Sigma}}_{p l}=\frac{\left(n_{c}-1\right) \hat{\boldsymbol{\Sigma}}_{c}+\left(n_{h}-1\right) \hat{\boldsymbol{\Sigma}}_{h}}{n_{c}+n_{h}-2}
$$

The $T^{2}$ statistic follows Hotelling's distribution, $T_{m, n_{c}+n_{h}-2}^{2}$, with $m$ and $n_{c}+n_{h}$-2 degrees-of-freedom, thus a statistical test for SDD can be defined as:

$$
\begin{array}{ccc}
T^{2} \leq F_{T_{m, n_{c}+n_{h}-2}^{-1}}^{-1}(1-\alpha) & \Rightarrow H_{0} \text { is accepted } \\
\text { Else } & \Rightarrow H_{0} \text { is rejected }
\end{array}
$$

where $F_{T_{m, n_{c}+n_{h}-2}^{2}}^{-1}(1-\alpha)$ is the $T_{m, n_{c}+n_{h}-2}^{2}$ inverse cumulative distribution function at a selected level of significance, $\alpha$.

However, this threshold cannot directly be used as objective function because it depends on the number of DSF components, $m$. Therefore, the SDD objective function, $J_{S D D}$, is defined as:

$$
J_{S D D}=T^{2} / F_{T_{m, n_{h}+n_{c-2}}^{-1}}^{2}(1-\alpha)
$$

where the $T^{2}$ statistic is standardized with respect to the corresponding, degree-of-freedom-dependent, statistical threshold $F_{T_{m, n_{c}+n_{h^{-2}}}^{-1}}^{2}(1-\alpha)$. Additionally, the accuracy, $A c c$, can be used to assess the performance of a classifier based on statistical hypothesis testing. It is defined ${ }^{20}$ as the sum of predicted true positives, $b^{+}$, and true negatives, $b^{-}$, divided by the total number of samples, $b_{t o t}$ :

$$
A c c=\left(b^{+}+b^{-}\right) / b_{t o t}
$$


Return to the Manage Active Submissions page at http://spie.org/submissions/tasks.aspx and approve or disapprove this submission. Your manuscript will not be published without this approval. Please contact author_help@spie.org with any questions or concerns.

\subsection{Statistical classification}

The problem of SDC is more complex and requires the definition of a set of $C_{D}$ competing hypotheses corresponding to $C_{D}$ damage classes as:

$$
\begin{array}{ccc}
H_{1}: & \boldsymbol{\mu}_{c}=\boldsymbol{\mu}_{1} & \text { (damage state 1) } \\
H_{2}: & \boldsymbol{\mu}_{c}=\boldsymbol{\mu}_{2} & (\text { damage state 2) } \\
\vdots & \vdots & \vdots \\
H_{C_{D}}: & \boldsymbol{\mu}_{c}=\boldsymbol{\mu}_{C_{D}} & \left(\text { damage state } C_{D}\right. \text { ) }
\end{array}
$$

where each hypothesis, $H_{s t}$, refers to a specific damage state, st, characterized by a mean DSF vector, $\boldsymbol{\mu}_{s t}$. From Bayes' theorem, the conditional posterior probability, $P\left(s t \mid \boldsymbol{\mu}_{c}\right)$, of a damage state $s t$ given a current DSF mean vector, $\boldsymbol{\mu}_{c}$, is ${ }^{19}$ :

$$
P\left(s t \mid \boldsymbol{\mu}_{c}\right)=\frac{p\left(\boldsymbol{\mu}_{c} \mid s t\right)}{\sum_{i=1}^{C_{D}} p\left(\boldsymbol{\mu}_{c} \mid i\right)}
$$

where $p\left(\boldsymbol{\mu}_{c} \mid i\right)$ is the conditional probability mass distribution of $\boldsymbol{\mu}_{c}$ given it belongs to the $i$-th damage state. It should be noted that the prior probabilities of the damage states and the corresponding cost functions are ignored in this study, but they could easily be included.

Although, multivariate Gaussian distributed variables are assumed, the probability densities $p\left(\boldsymbol{\mu}_{c} \mid i\right)$ are not readily available. According to Eq. (9), the probability of $\boldsymbol{\mu}_{c}$ belonging to the $i$-th damage state can be described with the help of the $T^{2}$ statistic. This approach allows using bins of samples to control the accuracy of the classification. However, the corresponding $T_{m, n_{i}+n_{c}-2}^{2}$ probability distribution function is rather complex and not commonly used. Therefore, $T^{2}$ statistic values are transformed into their $T_{F}^{2}$ counterparts that follow the more common $F$ distribution with $m$ and $n_{i}+n_{c}-1$ degrees-of-freedom as ${ }^{19}$ :

$$
T_{F}^{2}=\frac{n_{i}+n_{c}-m-1}{\left(n_{i}+n_{c}-2\right) m} T^{2} \square F_{m, n_{i}+n_{c}-m-1}
$$

Thus, the conditional probability densities, $p\left(\boldsymbol{\mu}_{c} \mid i\right)$, can be calculated as ${ }^{21}$ :

$$
p\left(\boldsymbol{\mu}_{c} \mid i\right)=\frac{\Gamma\left(\frac{m}{2}+\frac{n_{i}+n_{c}-1}{c}\right)}{\Gamma\left(\frac{m}{2}\right) \Gamma\left(\frac{n_{i}+n_{c}-1}{2}\right)}\left(\frac{m}{n_{i}+n_{c}-1}\right)^{\frac{m}{2}}\left(T_{F}^{2}\right)^{\frac{m}{2}-1}\left(1+\frac{m}{n_{i}+n_{c}-1} T_{F}^{2}\right)^{-\frac{m+n_{i}+n_{c}-1}{2}}
$$

where $\Gamma()$ denotes the Gamma function. The posterior probabilities in Eq. (15) can then be computed using conventional software packages, e.g. MATLAB ${ }^{22}$. The current damage state can be classified according to the maximum of these probabilities over all the $C_{D}$ damage states.

The SDC objective function, $J_{S D C}$, is defined by the ratio of correct classifications over the $C_{D}$ damage states as:

$$
J_{S D C}=\frac{1}{C_{D}} \sum_{i=1}^{C_{D}} \frac{b_{i}^{+}}{b_{i}^{*}}
$$

where $b_{i}^{*}$ and $b_{i}^{+}$are the true and the correctly classified number of bins of the $i$-th damage state training dataset, respectively. It measures the overall classification accuracy and is bounded between zero and one, where the latter corresponds to the perfect accuracy.

\section{PHYSICAL EXPERIMENTS}

\subsection{Experimental configuration}

The applicability of the proposed methodologies for SDD and SDC are assessed by means of physical experiments using a laboratory scale WTB of $2.36 \mathrm{~m}$ length. The WTB has a solid cross-section of $160 \mathrm{~mm}$ width made of a pultruded 
Return to the Manage Active Submissions page at http://spie.org/submissions/tasks.aspx and approve or disapprove this submission. Your manuscript will not be published without this approval. Please contact author_help@spie.org with any questions or concerns.

glass-fiber reinforced epoxy composite. The shape of the cross-section is defined by the airfoil E387, see Figure 1. The total mass of the WTB was measured as $7,110 \mathrm{~g}$ and the mass density is calculated based on the geometry as $2.30 \mathrm{~g} / \mathrm{cm}^{3}$. A vertical configuration was chosen for the dynamic experiments, as shown in Figure 2. Cantilever-type boundary conditions were realized by fixing the WTB root rigidly to a massive steel base sitting on a concrete floor. The WTB is excited without contact using a household pedestal fan with $40.6 \mathrm{~cm}$ rotor diameter and a maximum power output of 40 $\mathrm{W}$, where the second out of three selectable power levels was used. The fan was positioned $100 \mathrm{~cm}$ from the leadingedge of the WTB and its height was adjusted to $62.0 \mathrm{~cm}$ measured from the WTB root. This setup creates a wind-like airstream with an angle-of-attack of zero degrees typical for inspections of an idle WT. In this study, a minimal instrumentation setup was utilized, where only the flap-wise acceleration responses at the WTB tip were measured using a miniature piezoelectric accelerometer model Metra KS94B-100 with a sensitivity of approximately $100 \mathrm{mV} / \mathrm{g}$ and a frequency range between $0.5 \mathrm{~Hz}$ and $28 \mathrm{kHz}$. Signals were acquired with a National Instruments data acquisition card model NI-9234 connected to a National Instruments cDAQ-9174 chassis and a laptop with the National Instruments LabView software package.

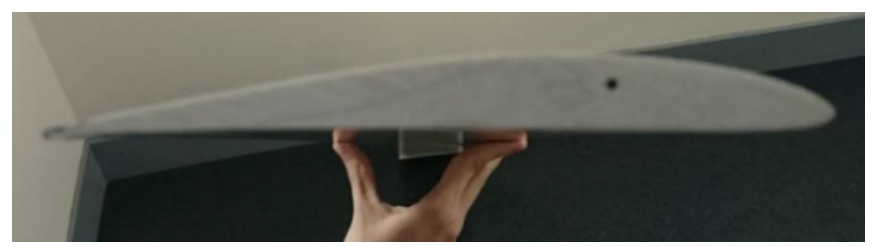

Figure 1. Cross-section of WTB.

Inspection reports and damage studies of WTBs identified damage hotspots at the trailing and leading edges at locations $30 \%$ and $70 \%$ of the blade length from the root and at the tip, respectibvely ${ }^{23-25}$. Therefore, damage was simulated nondestructively by attaching small masses at these locations, see Figure 2. Papatheou et al. introduced such pseudo-faults in SHM studies on a simple plate structure ${ }^{26}$ as well as on an aircraft wing ${ }^{27}$. The masses were varied between $10 \mathrm{~g}$ and $100 \mathrm{~g}$ in the different locations, as detailed in Table 1.

\subsection{Damage sensitive feature extraction}

The acceleration responses were acquired for the healthy and the damage states in sequences of 30 min length at a constant sampling rate of 2,048 Hz. Data processing and storage demands were reduced by low-pass filtering of the signals with an eight order Chebyshev type I filter with a cut-off frequency of $204.8 \mathrm{~Hz}$ and resampling at $256 \mathrm{~Hz}$. Each signal was then divided into 400 time series segments of a $5 \mathrm{~s}$ length with an overlap of approximately $10 \%$. To reduce effects of between-segment variations, the segments were normalized by removing their estimated means and dividing their estimated standard deviations.

In the next step, initial DSFs were extracted from the acceleration signal segments. PACCs from lag one to 100 were estimated from the healthy state signals. Figure 3 shows the estimated mean values of PACCs together with the statistical threshold of $95 \%$ confidence for PACCs to be significant. Although a clear cut-off cannot be identified, PACCs of lags above 80 fall mainly below the threshold. Thus, the initial DSF vectors were defined using all consecutive PACCs with lags one to 80 .

To reduce effects from varying ambient conditions, PCA was performed to transform the PACCs into DSFs with enhanced SDD and SDC properties. The PCA transformation matrix, T, was obtained by singular value decomposition of the PACC-based DSF's healthy state variance-covariance matrix. The variances explained by PCs are shown in Figure 4. It can be seen that the first 14 PCA scores contribute most to the total variance, while the variances of scores larger than 14 have only a minor contribution. Nevertheless, this does not allow directly selecting PCs with the best SDD and/or SDC performance. 
Return to the Manage Active Submissions page at http://spie.org/submissions/tasks.aspx and approve or disapprove this submission. Your manuscript will not be published without this approval. Please contact author_help@spie.org with any questions or concerns.

\section{STRUCTURAL DAMAGE DETECTION}

The proposed SDD methodology is based on the premise that including noisy or insensitive DSF components adversely affects the SHM performance, they should thus be eliminated from amongst useful the DSF. In this section, the FF selection is utilized for selecting optimal DSF subsets with respect to the SDD objective function, $J_{S D D}$, (Eq. (12)) using a $5 \%$ level of significance. The identification of optimal DSFs was done using the three healthy and eight damage datasets given in Table 1 with 400 DSF vector samples from each state. The result of the FF procedure is shown in Figure 5 for the PACCs and PCA scores. The initial features attain their maximum of $J_{S D D}=27.95$ using only the PACC at lag 17. Using four PCA scores $1,8,11$ and 13 outperforms the initial DSFs with an objective function value of $J_{S D D}=32.13$. The objective function for both DSFs decays towards the same minimum of 5.38 for the total number of available DSF components. Furthermore, all the $J_{S D D}$ values exceed unity, thus all presented damage scenarios are detectable using any set of initial or transformed DSFs.

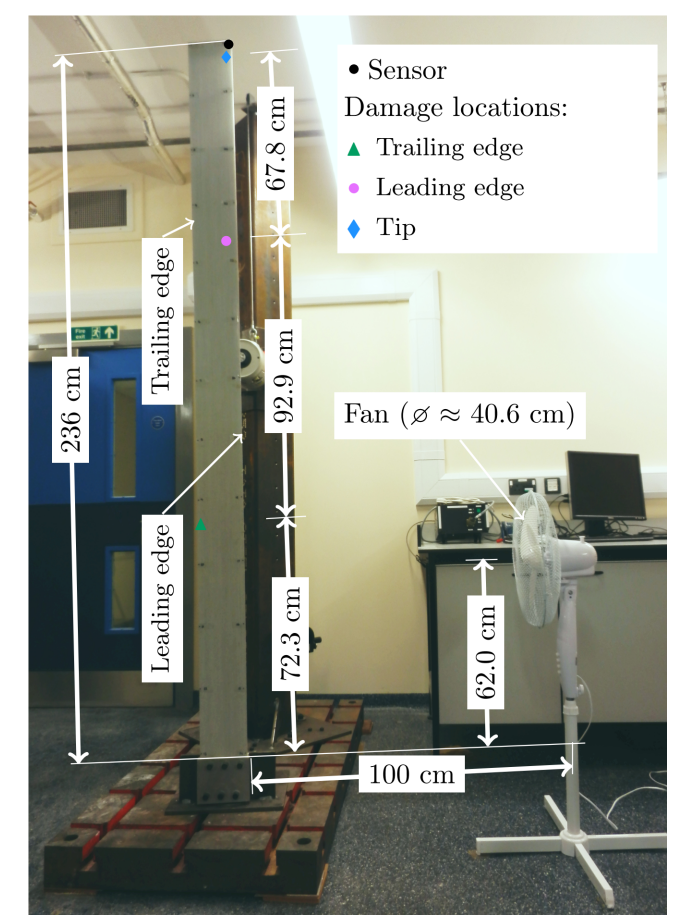

Figure 2. Experimental configuration.

Table 1. Non-destructive damage scenarios.

\begin{tabular}{|c|c|c|c|}
\hline No. & Name & Location & Extent \\
\hline 1 & TE-1 & Trailing edge, $72.3 \mathrm{~cm}$ from root & $20 \mathrm{~g}$ \\
\hline 2 & TE-2 & Trailing edge, $72.3 \mathrm{~cm}$ from root & $50 \mathrm{~g}$ \\
\hline 3 & TE-3 & Trailing edge, $2.3 \mathrm{~cm}$ from root & $80 \mathrm{~g}$ \\
\hline 4 & LE-1 & Leading edge, $165.2 \mathrm{~cm}$ from root & $20 \mathrm{~g}$ \\
\hline 5 & LE-2 & Leading edge, $165.2 \mathrm{~cm}$ from root & $50 \mathrm{~g}$ \\
\hline 6 & LE-3 & Leading edge, $165.2 \mathrm{~cm}$ from root & $80 \mathrm{~g}$ \\
\hline 7 & T-1 & Leading edge, $233.0 \mathrm{~cm}$ from root & $20 \mathrm{~g}$ \\
\hline 8 & T-2 & Leading edge, $233.0 \mathrm{~cm}$ from root & $50 \mathrm{~g}$ \\
\hline
\end{tabular}


Return to the Manage Active Submissions page at http://spie.org/submissions/tasks.aspx and approve or disapprove this submission. Your manuscript will not be published without this approval. Please contact author_help@spie.org with any questions or concerns.

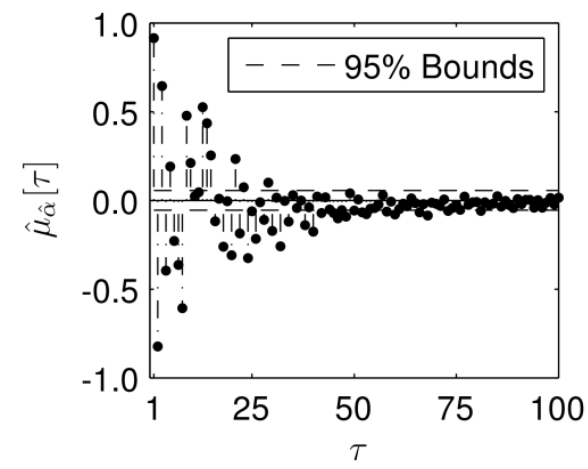

Figure 3. Estimated means of PACCs in healthy state.

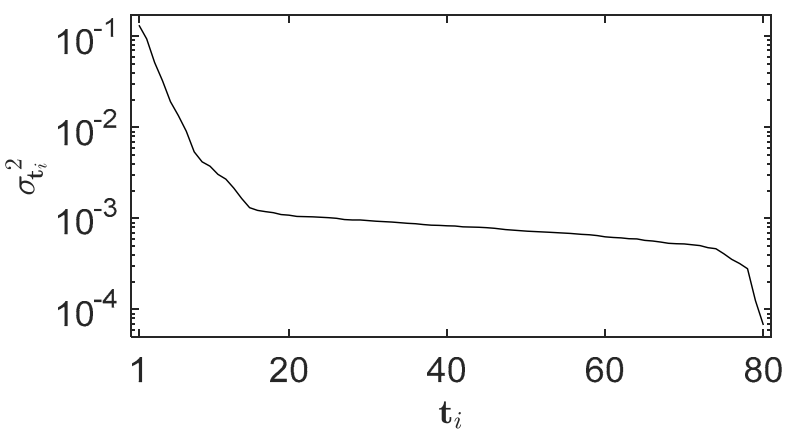

Figure 4. Variance explained by principal components of PACCs in healthy state.

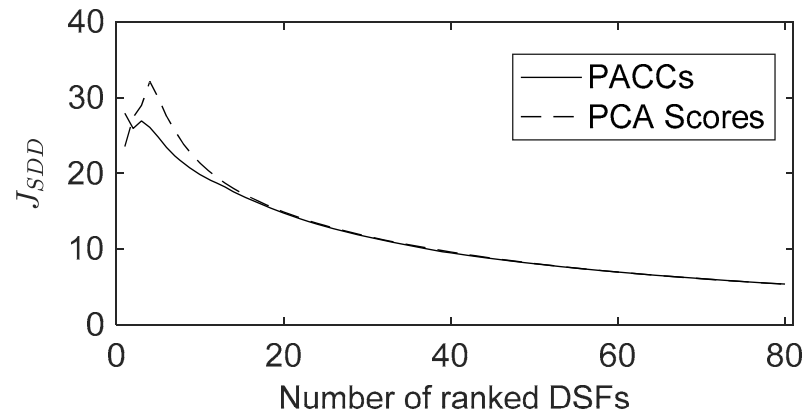

Figure 5. Fast forward ranking of PACCs and PCA scores for SDD.

Next, the identified optimal DSFs were employed for SDD on previously unseen datasets from the healthy and the eight damage states. The required statistical properties for calculating the $T^{2}$ statistics were estimated from the training dataset. The results are presented in Figure 6, where bins of the current state DSFs contain only single DSF samples. It can be seen that for the healthy state samples from both DSF types fall mainly below the detection threshold indicating acceptable numbers of false positives. Significant differences can be observed for the trailing edge damage scenarios, where PCA scores allow almost perfect detection of all three damage extents. For the same scenario, the selected PACC cannot detect damage of $20 \mathrm{~g}$ and $50 \mathrm{~g}$ and the largest damage extent of $80 \mathrm{~g}$ can only be partially detected. The results 
Return to the Manage Active Submissions page at http://spie.org/submissions/tasks.aspx and approve or disapprove this submission. Your manuscript will not be published without this approval. Please contact author_help@spie.org with any questions or concerns.

for the leading edge damages show more similarity between PACCs and PCA scores, but the PCA scores performed slightly better. This was especially the case for the $80 \mathrm{~g}$ damage, which could be well detected using the PCA scores. For the tip damages, the PCA scores allowed detecting the $50 \mathrm{~g}$ damage, while the PACCs could not detect this damage. These observations can be underpinned by the overall accuracies (Eq. (13)) of 0.45 and 0.73 achieved for the selected PACCs and PCA scores, respectively. The application of $T^{2}$ statistics allows using bins of samples in the current state in order to improve the accuracy. Bins of two and five samples increase the accuracy for the PACCs to 0.55 and 0.73 , respectively. However, the best performance can be achieved with five sample bins of PCA scores with a value of 0.95 followed by two sample bins with 0.86 , respectively.

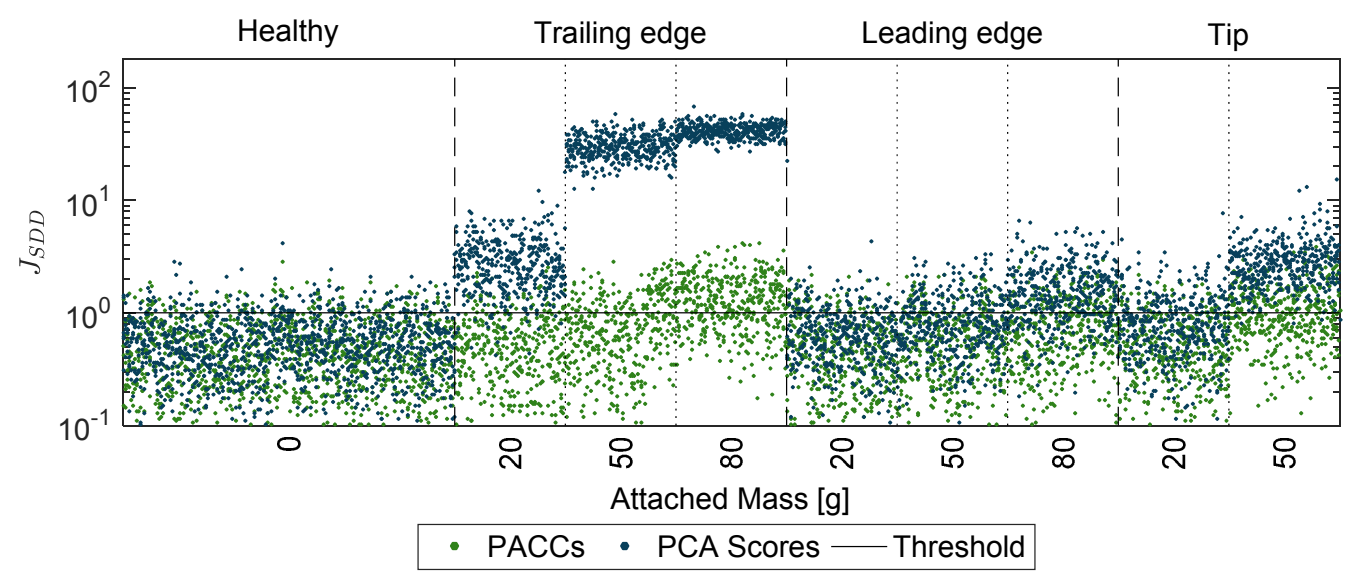

Figure 6. Detection results for selected PACCs and PCA scores using single samples of previously unseen datasets.

\section{STRUCTURAL DAMAGE CLASSIFICATION}

The risk that using all the available DSF components may lead to suboptimal results due to inclusion of noisy or insensitive components applies also to SDC. Therefore, DSFs are selected to maximize the SDC objective function, $J_{S D C}$, (Eq. (18)) in order to evaluate their sensitivity for this SHM task in terms of the average accuracy. The reference statistical properties of the damage classes specified in Table 1 were estimated using all the 400 DSF samples available from each class. The healthy state was not included because SDC will only be performed when the existence of damage has been indicated, e.g. with the help of statistical hypothesis testing as discussed earlier. To calculate the values of objective function $J_{S D C}$, bins of 20 consecutive DSF samples were drawn from the available DSF samples of each separate damage states. Using the full sets was not possible because the $T^{2}$ statistics would be zero and no information about the feature sensitivity could be obtained. The results of the FF procedure are shown in Figure 7 . The objective function value for using the best PCA score is 0.79 , i.e. approximately twice as high as for the best PACC with 0.44 . Adding more DSF components improves quickly the accuracies for both DSFs, but six PCA scores give already an objective function value of 0.94 outperforming the PACCs. Nevertheless, the overall best DSF selections led to similar results with accuracies of 0.97 and 0.98 for the PACCs and PCA scores, respectively. These selections outperform the full sets of available DSFs, which gave only an accuracy of 0.90 .

The performance of the DSF selections was investigated on previously unseen datasets of the eight damage scenarios with 400 samples drawn from each state. The referential statistical properties are estimated from the 400 DSF samples per damage class used in the training phase. To investigate the effect of the bin size in the current state classification, bins with one, two and five samples were studied and the relative rates of correct classifications are reported in Table 2 for each damage class. For both DSF types, the bins with five samples gave the best classification rates for each class. Nonetheless, the most significant improvements were achieved by doubling the samples from one to two. Furthermore, considerable differences can be observed between the damage scenarios, where the leading edge damages were the most difficult to classify followed by the damages at the trailing edge and the tip damage with $20 \mathrm{~g}$ attached mass. The remaining damages could be identified confidently even when bins with only one sample were used. A direct comparison 
Return to the Manage Active Submissions page at http://spie.org/submissions/tasks.aspx and approve or disapprove this submission. Your manuscript will not be published without this approval. Please contact author_help@spie.org with any questions or concerns.

of DSFs with identical bin sizes reveals that the PCA scores outperformed the PACCs in every case. This observation testifies to the benefits of the proposed optimal DSF selection methodology.

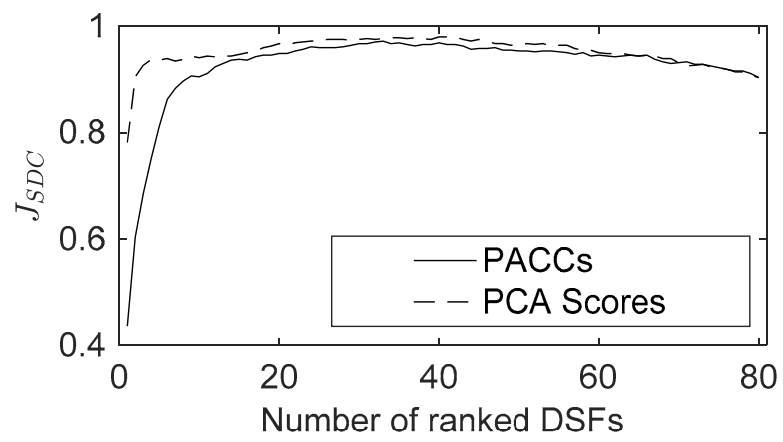

Figure 7. Fast forward ranking of PACCs and PCA scores for SDC.

Table 2. Relative number of correctly classified DSF bins using previously unseen datasets and different bin sizes.

\begin{tabular}{|c|c|c|c|c|c|c|c|}
\hline \multirow{2}{*}{ Damage } & \multicolumn{3}{|c|}{ PACCs } & \multicolumn{3}{c|}{ PCA Scores } \\
\cline { 2 - 8 } \multicolumn{2}{|c|}{} & \multicolumn{3}{|c|}{ Bin Size } & \multicolumn{3}{c|}{ Bin Size } \\
\hline No. & Name & 1 & 2 & 5 & 1 & 2 & 5 \\
\hline 1 & TE-1 & 0.53 & 0.81 & 0.96 & 0.87 & 0.98 & 1.00 \\
\hline 2 & TE-2 & 0.99 & 1.00 & 1.00 & 1.00 & 1.00 & 1.00 \\
\hline 3 & TE-3 & 1.00 & 1.00 & 1.00 & 1.00 & 1.00 & 1.00 \\
\hline 4 & LE-1 & 0.42 & 0.66 & 0.84 & 0.56 & 0.72 & 0.89 \\
\hline 5 & LE-2 & 0.24 & 0.32 & 0.36 & 0.32 & 0.42 & 0.51 \\
\hline 6 & LE-3 & 0.36 & 0.52 & 0.66 & 0.43 & 0.57 & 0.76 \\
\hline 7 & T-1 & 0.58 & 0.85 & 0.98 & 0.86 & 0.99 & 1.00 \\
\hline 8 & T-2 & 0.96 & 0.99 & 1.0 & 0.99 & 1.00 & 1.00 \\
\hline
\end{tabular}

\section{CONCLUSIONS}

This paper presents a methodology for statistical SDD and SDC featuring enhanced vibration-based DSFs and advanced statistics. DSFs were defined as PACCs and PCA scores from the PACCs extracted from acceleration responses. The method was applied to data gathered from experiments with a small scale WTB, where wind-like excitations were created with the help of a pedestal fan. Various damage extent scenarios were simulated non-destructively by attaching small masses at different locations.

The method was developed under the premise that initial multivariate DSFs will be composed of components with different sensitivities to damages and noise, therefore, including all the DSF components may lead to suboptimal SDD and SDC results. Therefore, DSF compositions were optimized using the FF selection algorithm and specialized objective functions for SDD and SDC. Binary statistical hypothesis testing was employed for SDD, thus a statistical distance measure was used in the SDD objective function. For SDC, a measure of the classification accuracy was utilized because damages are classified by a statistical classification approach based on Bayes' theorem. This approach also made use of an advanced statistic, which enabled improving the SDC performance by using bins of DSF samples. 
Return to the Manage Active Submissions page at http://spie.org/submissions/tasks.aspx and approve or disapprove this submission. Your manuscript will not be published without this approval. Please contact author_help@spie.org with any questions or concerns.

The expected benefits of the proposed methodologies were confirmed by the obtained results, where the DSF selections demonstrated an edge over the full sets of available DSFs. Furthermore, the optimally selected PCA scores outperformed the optimal selections of PACCs for SDD as well as for SDC. Finally, it could be demonstrated that the proposed SDD and SDC approached allowed classifying damage states with high accuracy using bins of DSFs samples extracted from signals of a single sensor. These results are promising for enhancing the ability to detect and classify damage scenarios with higher accuracy. This can pave the way for future applications in real WTBs.

\section{ACKNOWLEDGEMENT}

Simon Hoell's PhD study within the Lloyd's Register Foundation Centre for Safety and Reliability Engineering at the University of Aberdeen was supported by Lloyd's Register Foundation. The Foundation helps to protect life and property by supporting engineering-related education, public engagement and the application of research.

\section{REFERENCES}

[1] Jensen, F. M., Kling, A. and Sørensen, J. D., "Scale-Up of Wind Turbine Blades - Changes in Failure Type," EWEA Annual Event 2012 (EWEA 2012), 1-6 (2012).

[2] Det Norske Veritas (DNV), "Offshore Standard: DNV-OS-J101," (2011).

[3] Lloyd's Register, "Guidance on Offshore Wind Farm Certification," (2012).

[4] Blanco, M. I., "The economics of wind energy," Renew. Sustain. Energy Rev. 13(6-7), 1372-1382 (2009).

[5] Carden, E. P. and Fanning, P., "Vibration based condition monitoring: A review," Struct. Health Monit. 3(4), 355377 (2004).

[6] Hoell, S. and Omenzetter, P., "Optimal selection of autoregressive model coefficients for early damage detectability with an application to wind turbine blades," Mech. Syst. Signal Process. 70-71, 557-577 (2016).

[7] Kumar, R. P., Oshima, T., Mikami, S., Miyamori, Y. and Yamazaki, T., "Damage identification in a lightly reinforced concrete beam based on changes in the power spectral density," Struct. Infrastructure Eng. 8(8), 715-727 (2012).

[8] Roveri, N. and Carcaterra, A., "Damage detection in structures under traveling loads by Hilbert-Huang transform," Mech. Syst. Signal Process. 28, 128-144 (2012).

[9] Catbas, F. N., Gokce, H. B. and Gul, M., "Nonparametric analysis of structural health monitoring data for identification and localization of changes: Concept, lab, and real-life studies," Struct. Health Monit. 11(5), 613-626 (2012).

[10] Kay, S. M., "Modern Spectral Estimation: Theory and Application," Prentice Hall, Upper Saddle River, USA (1988).

[11] Liu, H. B. and Jiao, Y. B., "Application of genetic algorithm-support vector machine (GA-SVM) for damage identification of bridge," Int. J. Comput. Intell. Appl. 10(4), 383-397 (2011).

[12] Kim, Y., Chong, J. W., Chon, K. H. and Kim, J., "Wavelet-based AR-SVM for health monitoring of smart structures," Smart Mater. Struct. 22(1), 1-12 (2013).

[13] Bandara, R. P., Chan, T. H. T. and Thambiratnam, D. P., "Frequency response function based damage identification using principal component analysis and pattern recognition technique," Eng. Struct. 66, 116-128 (2014).

[14] de Lautour, O. R. and Omenzetter, P., "Nearest neighbor and learning vector quantization classification for damage detection using time series analysis," J. Struct. Control Health Monit. 17(6), 614-631 (2010).

[15] Hoell, S. and Omenzetter, P., "Improved damage detectability in a wind turbine blade by optimal selection of vibration signal correlation coefficients," Struct. Health Monit. 15(6), 685-705 (2016).

[16] Cryer, J. D. and Chan, K., "Time Series Analysis with Applications in R," Springer Science+Business Media, New York, USA (2008).

[17] Rasch, D., Pilz, J., Verdooren, R. and Gebhardt, A.,"Optimal Experimental Design with R," Taylor \& Francis, Boca Raton, USA (2011).

[18] Jolliffe, I. T., "Principal Component Analysis," Springer, New York, USA (2002).

[19] Rencher, A. C., "Multivariate Statistical Inference and Applications," John Wiley \& Sons, New York, USA (1998).

[20] Fawcett, T., "An introduction to ROC analysis," Pattern Recogn. Lett. 27(8), 861-874 (2006).

[21] Montgomery, D. C., "Introduction to Statistical Quality Control," John Wiley \& Sons, New York, USA (1997).

10171 - 12 V. 3 (p.11 of 12) / Color: No / Format: A4 / Date: 3/30/2017 2:46:29 AM

SPIE USE:

DB Check,

Prod Check, Notes: 
Return to the Manage Active Submissions page at http://spie.org/submissions/tasks.aspx and approve or disapprove this submission. Your manuscript will not be published without this approval. Please contact author_help@spie.org with any questions or concerns.

[22] The MathWorks, "MATLAB ${ }^{\circledR}, "(2014)$.

[23] Ataya, S. and Ahmed, M. M. Z., "Damages of wind turbine blade trailing edge: Forms, location, and root causes," Eng. Failure Anal. 35, 480-488 (2013).

[24] Ataya, S. and Ahmed, M. M. Z., "Forms of discontinuities in $100 \mathrm{~kW}$ and $300 \mathrm{~kW}$ Wind Turbine Blades," $10^{\text {th }}$ World Wind Energy Conference \& Renewable Energy Exhibition 2011, 1-6 (2011).

[25] Jensen, F. M., Sørensen, J. D., Nielsen, P. H., Berring, P. and Flores, S., "Failures in trailing edge bondlines of wind turbine blades," The 32nd Risø International Symposium on Materials Science, 319-327 (2011).

[26] Papatheou, E., Manson, G., Barthorpe, R. J. and Worden, K., "The use of pseudo-faults for novelty detection in SHM," J. Sound Vibrat. 329(12), 2349-2366 (2010).

[27] Papatheou, E., Manson, G., Barthorpe, R. J. and Worden, K., "The use of pseudo-faults for damage location in SHM: An experimental investigation on a Piper Tomahawk aircraft wing," J. Sound Vibrat. 333(3), 971-990 (2014). 